\title{
Six-year Optical Monitoring of BL Lacertae Object 1ES $0806+52.4$
}

\author{
Zhongyi Man, Xiaoyuan Zhang, and Jianghua Wu \\ Department of Astronomy, Beijing Normal University, Beijing 100875, China; \\ jhwu@bnu.edu.cn
}

$\mathrm{Xu}$ Zhou

Key Laboratory of Optical Astronomy, National Astronomical Observatories, Chinese Academy of Sciences, 20A Datun Road, Beijing 100012, China

Qirong Yuan

Department of Physics and Institute of Theoretical Physics, Nanjing Normal University, Nanjing 210046, China

Received __; accepted _ 


\begin{abstract}
We present the results of the first systematic long-term multi-color optical monitoring of the BL Lacertae object 1ES 0806+52.4. The monitoring was performed in multiple passbands with a 60/90 cm Schmidt telescope from December 2005 to February 2011. The overall brightness of this object decreased from 2005 December to 2008 December, and regained after that. A sharp outburst probably occurred around the end of our monitoring program. Overlapped on the long-term trend are some short-term small-amplitude oscillations. No intranight variability was found in the object, which is in accord with the historical observations before 2005. By investigating the color behavior, we found strong bluer-when-brighter chromatism for the long-term variability of 1ES 0806+52.4. The total amplitudes at the $c, i$, and $o$ bands are 1.18, 1.12, and 1.02 mags, respectively. The amplitudes tend to increase toward shorter wavelength, which may be the major cause of bluer-when-brighter. Such bluer-when-brighter is also found in other blazars like S5 0716+714, OJ 287, etc. The hard X-ray data collected from the Swift/BAT archive was correlated with our optical data. No positive result was found, the reason of which may be that the hard X-ray flux is a combination of the synchrotron and inverse Compton emission but with different timescales and cadences under the leptonic Synchrotron-Self-Compton (SSC) model.
\end{abstract}

Subject headings: BL lacertae Object: individual (1ES 0806+524) — galaxies: active -galaxies: photometry 


\section{Introduction}

Active galactic nuclei (AGN) is powered by a supermassive black hole surrounded by an accretion disk. Blazar, one of the most violently variable class of AGN, is distinguished by large and rapid flux variability (Bonning et al.|2012; Sandrinelli et al.|2014), high and variable polarization (Hagen-Thorn et al. 2008; Sasada et al. 2008; Gaur et al. 2014), and basically a non-thermal continuum. Depending on the presence of strong emission lines in spectrum, a blazar can be further termed either as a flat-spectrum radio quasar (FSRQ) or a BL Lacerate object.

Two typical components can be seen in the spectral energy distribution (SED) of blazars: One at low frequencies from radio to UV or soft X-rays, the other ranges from X-rays to gamma-rays (e.g. Takahashi 2001; Bonning et al. 2012). The low energy component is believed to be caused by the synchrotron process of the relativistic particles in the jet. The high energy one, however, is interpreted as either due to inverse Compton scattering of the low energy photons by the same ensemble of relativistic electrons responsible for the synchrotron emission named SynchrotronSelf-Compton (SSC) model (e.g. Mücke et al. 2003; Krawczynski et al. 2002), or due to the hadronic processes initiated by synchrotron radiation of protons co-accelerated with the electrons in the jet (hardronic models e.g. Mücke \& Protheroe 2001; Levinson 2006).

1ES 0806+52.4 was identified as a BL Lac object (Schachter et al. 1993), based on the observations carried out on both radio band from the Green Bank $91 \mathrm{~m}$ telescope (Becker et al. 1991) and X-ray bands from the Einstein Slew Survey (Elvis et al. 1992). The redshift of its host galaxy is estimated as $z=0.138$ (Bade et al. 1998). Very high energy gamma-ray observations of 1ES $0806+52.4$ was reported by the Whipple Collaboration (de la Calle Pérez et al. 2003; Horan et al.|2004), the HEGRA Collaboration (Aharonian et al. 2004) and VERITAS (Weekes et al. 2002). R-band optical observations 
of this BL Lac object were conducted from 2000 to 2001 (Kurtanidze et al. 2002), and from 2002 to 2004 (Kapanadze 2009). The former highlighted the variability with one magnitude on long time scales (Kurtanidze et al. 2002). Till now, no long-term systematic optical monitoring has been conducted on this object, and no research has been made on the correlation between radiation at different wavelengths.

Our quasi-simultaneous multi-color monitoring of 1ES 0806+52.4 was carried out from 2005 December to 2011 February. In order to investigate the correlation between the optical and the hard X-ray emission, X-ray data covering the same period were collected from the Swift/BAT data archive and were analyzed with the optical data.

\section{Observation and Data Reduction}

Our observations were performed on a 60/90 cm Schmidt telescope at the Xinglong Station of the National Astronomical Observatories of China (NAOC). Mounted at the telescope main focus was a Ford Aerospace $2048 \times 2048$ thick CCD camera which was then surrogated by a new E2V 4096× 4096 CCD in 2006 December. The field of view is enlarged from $58^{\prime} \times 58^{\prime}$ to $96^{\prime} \times 96^{\prime}$. The resolution is increased from $1 .^{\prime \prime} 7 \mathrm{pixel}^{-1}$ to $1 .^{\prime \prime} 3 \mathrm{pixel}^{-1}$. The blue quantum efficiency of the new E2V thinned CCD is also higher than the former thick one. The telescope is equipped with a 15 color intermediate-band filters, covering a wavelength range from 3000 to 10,000 $\AA$ (Fan et al. 1996). Four F sub-dwarfs (Oke \& Gunn 1983) are used as the standard stars to calibrate the magnitude. For the details of the filter system, definition of the corresponding magnitudes and how to derive flux from the magnitude, one can refer to Yan et al. (2000); Zhou et al. (2001, 2003).

Our monitoring program on 1ES 0806+52.4 started on the night of 2005 December $12^{\text {th }}$, and ended on 2011 February $11^{\text {th }}$. After ruling out the nights with bad weather 
condition and those for other targets, the actual number of observation nights is 77 . Filters in $e, i$, and $m$ bands were adopted in 2005 - 2006. During most of the nights in this period, more than ten exposures, sometimes up to twenty, were made in each band with a temporal resolution about 20 minutes. Filters were then surrogated by $c$, $i$, and $o$ bands from the end of 2006 December to 2011 February, and less than ten exposures were made on most nights. The exposure time determined by the moon phase, weather condition and filter varies from 60 to 540 seconds. The central wavelengths of $c, e, i, m$, and $o$ bands are 4210, 4920, 6660, 8020, and $9190 \AA$, respectively. The $i$ magnitude can be transformed into the broadband R magnitude for stars by using the formula given by Zhou et al. (2003) and for AGNs by using the formula given by Dai et al. (2013).

We did bias subtraction and flat-fielding using the BATC automatic data reduction software PIPELINE I (Fan et al. 1996; Zheng et al.|1999). Four stars, C1, C2, C3, and C4 (see Fig. 1), were selected as reference stars (Fiorucci et al. 1998), and we chose star C5 as check star (for a reasonable selection of reference and check stars, see Howell et al. 1988). We extracted the instrumental aperture magnitudes of the blazar and the five comparison stars from each frame by using the algorithm of Stetson (1987). The radii of the aperture and the sky annuli were adopted as 3, 7, and 10 pixels, respectively, and they may be adjusted slightly according to the seeing. The brightness of the blazar was measured relative to the average magnitude of the four reference stars. The accuracy of our measurement is shown by the check star's differential magnitude, the difference between the magnitude of C5 and the average magnitude of the four reference stars. We observed stars C1, C2, C3, C4, C5 on a photometric night, and used the photometric F-type standard stars mentioned above to calibrate their standard magnitudes. The results are listed in Table 1. 


\section{Light Curve}

The samples of observational log and results are given in Table 2 - 6. The columns are observation date and time in universal time, exposure time in seconds, Julian date, magnitude, error, and differential magnitude of star C5 (its nightly averages were set to zero).

We gathered 1399 optical data points in total: numbers of photometric points for $c$, $e, i, m$, and $o$ bands are 292, 150,490, 171 and 296, respectively. The light curves of the overall monitoring period in five bands are shown in Figure 2. There are basically two outbursts with a concave shape between them. The peak of the first outburst occurred on 2006 Mar $15^{\text {th }}$ (JD 2,453,810), and then the brightness of the object started falling until 2008 Dec $11^{\text {th }}$ (JD 2,454,812). Later on, the curves turned up to the second outburst which is more powerful than the first one. No details can be known about the strong outburst because our observation ceased on 2011 Feb 11 th (JD 2,455,604). Overlapped on the long-term large-amplitude variations are some short-term small-amplitude oscillations during JD 2,454,000 to JD 2,455,000. We also notice that a double-peak structure of 1ES 0806+52.4 was spotted during 2002-2004 (Kapanadze 2009), yet it was not found in our observations.

The overall amplitudes in $e, i$, and $m$ bands from 2005 December $15^{\text {th }}$ to 2006 November $19^{\text {th }}$ are $0.17,0.12$, and 0.09 mags, respectively, the amplitudes in $c$, $i$, and $o$ band from 2006 December $4^{\text {th }}$ to 2011 February $11^{\text {th }}$ are $1.18,1.12$, and 1.02 mags. We note that the overall amplitudes tend to decline with the decreasing frequency. We examined the intra-night variability of this object by using the methods suggested by de Diego (2010), but eventually found no obvious intra-night variability during the whole time span. It is in accord with the observation from 2002 to 2004 (Kapanadze 2009), which detected no intra-night variability for this source either. Nevertheless, our average observational duration on each night is only 
3.6 hours, which may not be long enough to detect the intra-night variability, if there are some cases. Some blazars display strong intra-night variability. For example, S5 0716+714 was observed to vary by 0.117 mags in $1.1 \mathrm{hrs}$ (Dai et al. 2013). PKS 2155-304 was detected a very fast variability rate of $0.43 \mathrm{mag} / \mathrm{h}$ in the observation session (Sandrinelli et al. 2014).

\section{Color Behavior}

The long-term color behavior of 1ES 0806+52.4 was investigated based on our data. We adopted the $e$ and $m$ bands to calculate the color in the first stage from 2005 to 2006, and used the $c$ and $o$ bands in the second stage from 2006 to 2011. It is because the differences in wavelength of the two pairs of filters are relatively large, and can thereby denote a better spectral shape. The color-magnitude diagrams are displayed in Figure 3.

The linear least square method with measurement errors taken into consideration was used to fit the points. The points in the left panel for the first stage have a linear regression of $y=0.537 x-7.847$, and the correlation coefficient is 0.808 , which means a significance level of $95 \%$. The points in the right panel for the second stage have a linear regression of $y=0.176 x-1.791$, and the correlation coefficient is 0.792 , which also means a significance level of 95\%. Both panels show strong bluer-when-brighter (BWB) chromatism. The intra-night color behavior of 1ES $0806+52.4$ was not investigated due to the absence of intra-night variability.

A number of BL Lac objects have been reported displaying the BWB phenomenon so far. Significant BWB correlations were found for both inter-night and intra-night variations in the typical BL Lac object S5 0714+716 (e.g. Wu et al. 2005). Inter-night BWB was found in BL Lacerate (e.g. Zhai \& Wei 2012). In OJ 287, a well studied BL Lac, BWB is also found on long time scale (Dai et al. 2011). In fact, such BWB chromatism is likely to be a 
general feature of BL Lac objects (e.g. Vagnetti et al. 2003; Rani et al. 2010). For FSRQ, there were once claims of redder-when-brighter trend (e.g. Gu et al. 2006; Hu et al. 2006). However, Gu \& Ai (2011) found this color behavior for only one object in a sample of 29 FSRQs. The mechanism of BWB was simulated (Dai et al. 2011), and the result shows that BWB is probably due to the difference of the amplitude at different wavelengths. The variation amplitude of 1ES 0806+52.4 was found to decrease with decreasing frequency, which can at least partly explain the BWB behaviour observed in this object.

\section{Cross-correlation Analysis and Discussions}

In order to research the emission at different wavelengths, our optical data was compared with the time series data provided by Swift/Burst Alert Telescope (BAT), which has monitored 1ES 0806+52.4 in hard X-ray band since 2005. The Swift/BAT hard X-ray transient monitor provides near real-time coverage of the X-ray sky in the energy range $15-50 \mathrm{keV}$, or $3.63 \times 10^{18}-1.21 \times 10^{19} \mathrm{~Hz}$ in frequency. The BAT observes $88 \%$ of the sky each day with a detection sensitivity of 5.3 mCrab for a full-day observation and a time

resolution as fine as 64 seconds (Krimm et al. 2013). We used the daily average data offered by Swift/BAT. Both sequences were transformed into flux so that we can analyze them together. The light curves of i band and X-ray band are shown in Figure 4.

We used the z-transformed discrete correlation functions (ZDCFs; Alexander 1997) to search for the optical-hard X-ray correlations. The ZDCF differs from the discrete correlation function (DCF; Edelson \& Krolik 1988) for it bins the data points into equal population bins and uses Fishers z-transform to stabilize the highly skewed distribution of the correlation coefficient. It is much more efficient than the DCF in uncovering correlations involving the variability timescale, and deals with under-sampled light curves better than both the DCF and the interpolated cross-correlation function (ICCF; Gaskell \& Peterson 
1987). The results are displayed in Figure 5 for all the data from JD 2,453,717 to JD $2,455,604$.

In Figure 5, no obvious peak of the DCF values can be found, indicating an absence of correlation between the two bands. We try to explain the result as follows:

Firstly, there are daily data for X-ray band, yet the optical observation has not been taken regularly: 77 daily average points in 1187 days in total. The time resolution of our optical data is not compatible with that of Swift/BAT, which may possibly result in the loss of important fluctuations in the optical light curve, especially at the end of our monitoring program. It is probably hard to find a strong correlation between two samples with very different sampling rates. Secondly, as shown in Figure 4, no significant flare or outburst can be found in both optical and X-ray bands (There may be a strong flare at the end of the optical data but the measurements are too few. The X-ray data obviously have no corresponding flare). As a result, it might be hard to find any correlation.

Thirdly, provided that the no-correlation result is not caused by the limited data sampling in optical band or the lack of significant variability event in both bands, then it could be a reflect of a complex radiation mechanism. We notice that the hard X-ray band from $3.63 \times 10^{18} \mathrm{~Hz}$ to $1.21 \times 10^{19} \mathrm{~Hz}$ lies in the transition area of the two peaks in the SED of 1ES 0806+52.4 (Cogan \& VERITAS Collaboration 2008). Emission in this regime may be a combination of the synchrotron and inverse Compton mechanisms. Variations from the synchrotron and inverse Compton processes may have different amplitudes and cadences, hence a combination of them may show complicated variability and can hardly be found correlated with the optical variation. A similar explanation was raised to frame why the correlation between X-ray and other bands cannot be found in blazar S5 0716+714 (Rani et al. 2013).

Besides, there are also some other blazars showing no correlation between X-ray 
and other bands, which might be caused by the different location of emission regions in different bands. For instance, 3C 279 was observed by Abdo et al. (2010), and its X-ray emission has not been found accompanied quasi-simultaneously by the optical/GeV flares. An argument is that X-ray photons are produced further down to the jet compared to optical $\mathrm{GeV}$ photons. The case that the variations in X-ray band is not correlated with that of optical/gamma-ray (so-called orphan gamma-ray flare) band was also found in other blazars such as Mrk 501 (Neronov et al. 2012), 1ES 1959+650 (Daniel et al. 2005), etc. The TeV flare in Mrk 501 may be produced by an electromagnetic cascade initiated by very-high-energy gamma-rays in the intergalactic medium (Neronov et al. 2012), and a Hadronic Synchrotron Mirror Model was employed to explain the orphan TeV flare of 1ES

1959+650 (Daniel et al. 2005). These results, together with ours, can be used to investigate the detail of the variability of blazars, i.e. the radiation mechanism, the location of the emission region, etc.

\section{Conclusions}

Our monitoring work targeting at the BL Lac object 1ES 0806+52.4 was conducted in five intermediate optical wavebands from 2005 December to 2011 February using the 60/90 cm Schmidt telescope located at the Xinglong Station of the National Astronomical Observatories of China (NAOC). It was the first systematic multi-color optical monitoring of 1ES $0806+52.4$ on long timescale, and could thereby be effectively used to further study both long and short term flux and spectral variability of this object.

The analysis of the data reveals its overall brightness declined from 2005 December to 2008 December, and it turned brighter during 2009 to 2011. Overlapped on the long-term trend are some short-term small-amplitude oscillations. Unfortunately, our data samples are not abundant considering the long period of time we covered, and there are several 
interruptions in our observation. No obvious intra-night variability was found during the monitoring period, and the historical observation from 2002-2004 revealed the same feature. We did not find the double-peak structure as the former observation has suggested. Color behavior on long timescale was studied, and strong BWB phenomenon is revealed. This is consistent with the color behavior of most blazars. No obvious correlation was found between our optical variations and hard X-ray variability collected from the Swift/BAT archive. This result can be attributed to the different time resolution of the optical and X-ray bands or lack of significant variability event in the observation session; moreover, it may suggest the hard X-ray emission is a combination of both synchrotron radiation and inverse Compton scattering.

We gathered 1399 data points in total for 1ES 0806+52.4, which is the largest optical multi-color database for the variability of the object todate. Our data can also be correlated with the data other than the hard X-ray wavelength, e.g., radio, Gamma-ray, etc. for a more comprehensive investigation of the broad-band behavior of 1ES 0806+52.4.

We thank the anonymous referee for insightful comments and constructive suggestions, and we also thank Yang Chen for his advice and help to us. Our work has been supported by National Basic Research Program of China 973 Program 2013CB834900, Chinese National Natural Science Foundation grant 11273006, 11173016, 11073023 and the Scientific Research Foundation of Beijing Normal University. 


\section{REFERENCES}

Abdo, A. A., Ackermann, M., Ajello, M., et al. 2010, Nature, 463, 919

Aharonian, F., Akhperjanian, A., Beilicke, M., et al. 2004, A\&A, 421, 529

Alexander, T. 1997, in Astrophysics and Space Science Library, Vol. 218, Astronomical Time Series, ed. D. Maoz, A. Sternberg, \& E. M. Leibowitz, 163

Bade, N., Beckmann, V., Douglas, N. G., et al. 1998, A\&A, 334, 459

Becker, R. H., White, R. L., \& Edwards, A. L. 1991, ApJS, 75, 1

Bonning, E., Urry, C. M., Bailyn, C., et al. 2012, ApJ, 756, 13

Cogan, P., \& VERITAS Collaboration. 2008, in American Institute of Physics Conference Series, Vol. 1085, American Institute of Physics Conference Series, ed. F. A. Aharonian, W. Hofmann, \& F. Rieger, 403

Dai, Y., Wu, J., Zhu, Z.-H., Zhou, X., \& Ma, J. 2011, AJ, 141, 65

Dai, Y., Wu, J., Zhu, Z.-H., et al. 2013, ApJS, 204, 22

Daniel, M. K., Badran, H. M., Bond, I. H., et al. 2005, ApJ, 621, 181

de Diego, J. A. 2010, AJ, 139, 1269

de la Calle Pérez, I., Bond, I. H., Boyle, P. J., et al. 2003, ApJ, 599, 909

Edelson, R. A., \& Krolik, J. H. 1988, ApJ, 333, 646

Elvis, M., Plummer, D., Schachter, J., \& Fabbiano, G. 1992, ApJS, 80, 257

Fan, X., Burstein, D., Chen, J.-S., et al. 1996, AJ, 112, 628

Fiorucci, M., Tosti, G., \& Rizzi, N. 1998, PASP, 110, 105 
Gaskell, C. M., \& Peterson, B. M. 1987, ApJS, 65, 1

Gaur, H., Gupta, A. C., Wiita, P. J., et al. 2014, ApJ, 781, L4

Gu, M. F., \& Ai, Y. L. 2011, A\&A, 534, A59

Gu, M. F., Lee, C.-U., Pak, S., Yim, H. S., \& Fletcher, A. B. 2006, A\&A, 450, 39

Hagen-Thorn, V. A., Larionov, V. M., Jorstad, S. G., et al. 2008, ApJ, 672, 40

Horan, D., Badran, H. M., Bond, I. H., et al. 2004, ApJ, 603, 51

Howell, S. B., Warnock, III, A., \& Mitchell, K. J. 1988, AJ, 95, 247

Hu, S. M., Zhao, G., Guo, H. Y., Zhang, X., \& Zheng, Y. G. 2006, MNRAS, 371, 1243

Kapanadze, B. Z. 2009, MNRAS, 398, 832

Krawczynski, H., Coppi, P. S., \& Aharonian, F. 2002, MNRAS, 336, 721

Krimm, H. A., Holland, S. T., Corbet, R. H. D., et al. 2013, ApJS, 209, 14

Kurtanidze, O. M., Nikolashvili, M. G., Richter, G. M., \& Sigua, L. A. 2002, in X-ray Spectroscopy of AGN with Chandra and XMM-Newton, ed. T. Boller, S. Komossa, S. Kahn, H. Kunieda, \& L. Gallo, 301

Levinson, A. 2006, International Journal of Modern Physics A, 21, 6015

Mücke, A., \& Protheroe, R. J. 2001, Astroparticle Physics, 15, 121

Mücke, A., Protheroe, R. J., Engel, R., Rachen, J. P., \& Stanev, T. 2003, Astroparticle Physics, 18, 593

Neronov, A., Semikoz, D., \& Taylor, A. M. 2012, A\&A, 541, A31

Oke, J. B., \& Gunn, J. E. 1983, ApJ, 266, 713 
Rani, B., Gupta, A. C., Strigachev, A., et al. 2010, MNRAS, 404, 1992

Rani, B., Krichbaum, T. P., Fuhrmann, L., et al. 2013, A\&A, 552, A11

Sandrinelli, A., Covino, S., \& Treves, A. 2014, A\&A, 562, A79

Sasada, M., Uemura, M., Arai, A., et al. 2008, PASJ, 60, L37

Schachter, J. F., Stocke, J. T., Perlman, E., et al. 1993, ApJ, 412, 541

Stetson, P. B. 1987, PASP, 99, 191

Takahashi, T. 2001, in American Institute of Physics Conference Series, Vol. 558, American Institute of Physics Conference Series, ed. F. A. Aharonian \& H. J. Völk, 346-357

Vagnetti, F., Trevese, D., \& Nesci, R. 2003, ApJ, 590, 123

Weekes, T. C., Badran, H., Biller, S. D., et al. 2002, Astroparticle Physics, 17, 221

Wu, J., Peng, B., Zhou, X., et al. 2005, AJ, 129, 1818

Yan, H., Burstein, D., Fan, X., et al. 2000, PASP, 112, 691

Zhai, M., \& Wei, J. Y. 2012, A\&A, 538, A125

Zheng, Z., Shang, Z., Su, H., et al. 1999, AJ, 117, 2757

Zhou, X., Jiang, Z.-J., Xue, S.-J., et al. 2001, Chinese J. Astron. Astrophys., 1, 372

Zhou, X., Jiang, Z., Ma, J., et al. 2003, A\&A, 397, 361 
Table 1. Magnitude of 5 Comparison Stars.

\begin{tabular}{rccccc}
\hline \hline \multirow{2}{*}{ band } & $\mathrm{C} 1$ & $\mathrm{C} 2$ & $\mathrm{C} 3$ & $\mathrm{C} 4$ & $\mathrm{C} 5$ \\
\hline & & & & & \\
$c$ & 14.035 & 15.410 & 15.379 & 16.498 & 16.187 \\
$e$ & 13.291 & 14.859 & 14.938 & 15.901 & 15.809 \\
$i$ & 12.653 & 14.349 & 14.534 & 15.412 & 15.548 \\
$m$ & 12.476 & 14.216 & 14.420 & 15.245 & 15.256 \\
$o$ & 12.387 & 14.164 & 14.402 & 15.170 & 15.399 \\
\hline
\end{tabular}

Table 2. Observational Log and Results in the $c$ Band

\begin{tabular}{ccccccc}
\hline \hline & & & & & & \\
Observation Date & Observation Time & Exposure Time & Julian Date & $c$ & $c_{\text {err }}$ & dfmagC5 \\
$(\mathrm{UT})$ & $(\mathrm{UT})$ & $(\mathrm{s})$ & $\mathrm{JD}$ & $(\mathrm{mag})$ & $(\mathrm{mag})$ & $(\mathrm{mag})$ \\
\hline & & & & & & \\
20061204 & $18: 44: 51.0$ & 480 & 2454074.28115 & 15.886 & 0.034 & 0.064 \\
20061204 & $19: 07: 27.0$ & 480 & 2454074.29684 & 15.806 & 0.035 & -0.011 \\
20061204 & $19: 29: 52.0$ & 480 & 2454074.31241 & 15.809 & 0.031 & 0.008 \\
20061204 & $19: 52: 18.0$ & 480 & 2454074.32799 & 15.832 & 0.032 & 0.012 \\
20061204 & $20: 14: 53.0$ & 480 & 2454074.34367 & 15.850 & 0.037 & -0.006 \\
\hline
\end{tabular}

Note. - This table is available in its entirety in machine-readable and Virtual Observatory (VO) forms in the online journal. A portion is shown here for guidance regarding its form and content. 
Table 3. Observational Log and Results in the $e$ Band

\begin{tabular}{ccccccc}
\hline \hline $\begin{array}{c}\text { Observation Date } \\
(\mathrm{UT})\end{array}$ & $\begin{array}{r}\text { Observation Time } \\
(\mathrm{UT})\end{array}$ & $\begin{array}{r}\text { Exposure Time } \\
(\mathrm{s})\end{array}$ & $\begin{array}{c}\text { Julian Date } \\
\text { JD }\end{array}$ & $\begin{array}{c}e_{\text {err }} \\
(\mathrm{mag})\end{array}$ & $\begin{array}{c}\text { dfmagC5 } \\
(\mathrm{mag})\end{array}$ & $\begin{array}{c}\mathrm{mag}) \\
20051215\end{array}$ \\
\hline & $15: 01: 46.0$ & 300 & 2453720.12623 & 15.991 & 0.085 & -0.024 \\
20051215 & $15: 17: 43.0$ & 300 & 2453720.13730 & 15.993 & 0.078 & 0.106 \\
20051215 & $15: 34: 01.0$ & 300 & 2453720.14862 & 15.925 & 0.081 & -0.056 \\
20051215 & $15: 49: 56.0$ & 300 & 2453720.15968 & 15.894 & 0.083 & -0.053 \\
20051215 & $16: 05: 54.0$ & 300 & 2453720.17076 & 15.865 & 0.071 & -0.071 \\
\hline
\end{tabular}

Note. - This table is available in its entirety in machine-readable and Virtual Observatory (VO) forms in the online journal. A portion is shown here for guidance regarding its form and content.

Table 4. Observational Log and Results in the $i$ Band

\begin{tabular}{crrrrrr}
\hline \hline $\begin{array}{c}\text { Observation Date } \\
(\mathrm{UT})\end{array}$ & $\begin{array}{r}\text { Observation Time } \\
(\mathrm{UT})\end{array}$ & $\begin{array}{r}\text { Exposure Time } \\
(\mathrm{s})\end{array}$ & $\begin{array}{c}\text { Julian Date } \\
\text { JD }\end{array}$ & $\begin{array}{c}i \\
(\mathrm{mag})\end{array}$ & $\begin{array}{c}i_{\text {err }} \\
(\mathrm{mag})\end{array}$ & $\begin{array}{c}\text { dfmagC5 } \\
(\mathrm{mag})\end{array}$ \\
\hline & & & & & & \\
20051212 & $15: 44: 16.0$ & 180 & 2453717.15574 & 15.488 & 0.034 & -0.021 \\
20051212 & $15: 55: 15.0$ & 180 & 2453717.16337 & 15.514 & 0.033 & 0.006 \\
20051212 & $16: 05: 08.0$ & 180 & 2453717.17023 & 15.408 & 0.030 & -0.004 \\
20051212 & $16: 15: 18.0$ & 180 & 2453717.17729 & 15.416 & 0.030 & -0.024 \\
20051212 & $16: 25: 24.0$ & 180 & 2453717.18431 & 15.408 & 0.028 & 0.042 \\
\hline
\end{tabular}

Note. - This table is available in its entirety in machine-readable and Virtual Observatory (VO) forms in the online journal. A portion is shown here for guidance regarding its form and content. 
Table 5. Observational Log and Results in the $m$ Band

\begin{tabular}{|c|c|c|c|c|c|c|}
\hline $\begin{array}{l}\text { Observation Date } \\
\qquad(\mathrm{UT})\end{array}$ & $\begin{array}{l}\text { Observation Time } \\
\qquad(\mathrm{UT})\end{array}$ & $\begin{array}{c}\text { Exposure Time } \\
(\mathrm{s})\end{array}$ & $\begin{array}{c}\text { Julian Date } \\
\text { JD }\end{array}$ & $\begin{array}{c}m \\
(\mathrm{mag})\end{array}$ & $\begin{array}{l}m_{\text {err }} \\
(\mathrm{mag})\end{array}$ & $\begin{array}{c}\text { dfmagC5 } \\
\text { (mag) }\end{array}$ \\
\hline 20051212 & $15: 49: 15.0$ & 300 & 2453717.15920 & 15.248 & 0.044 & 0.014 \\
\hline 20051212 & $16: 00: 10.0$ & 300 & 2453717.16678 & 15.238 & 0.041 & -0.057 \\
\hline 20051212 & $16: 10: 21.0$ & 300 & 2453717.17385 & 15.189 & 0.040 & 0.053 \\
\hline 20051212 & $16: 20: 26.0$ & 300 & 2453717.18086 & 15.236 & 0.045 & -0.019 \\
\hline 20051212 & $16: 30: 24.0$ & 300 & 2453717.18778 & 15.264 & 0.051 & -0.006 \\
\hline
\end{tabular}

Note. - This table is available in its entirety in machine-readable and Virtual Observatory (VO) forms in the online journal. A portion is shown here for guidance regarding its form and content.

Table 6. Observational Log and Results in the $o$ Band

\begin{tabular}{ccccccc}
\hline \hline $\begin{array}{c}\text { Observation Date } \\
(\mathrm{UT})\end{array}$ & $\begin{array}{c}\text { Observation Time } \\
(\mathrm{UT})\end{array}$ & $\begin{array}{r}\text { Exposure Time } \\
(\mathrm{s})\end{array}$ & $\begin{array}{c}\text { Julian Date } \\
\text { JD }\end{array}$ & $\begin{array}{c}o \\
(\mathrm{mag})\end{array}$ & $\begin{array}{c}o_{\text {err }} \\
(\mathrm{mag})\end{array}$ & $\begin{array}{c}\text { dfmagC5 } \\
(\mathrm{mag})\end{array}$ \\
\hline & & & & & & \\
20061204 & $18: 57: 10.0$ & 480 & 2454074.28970 & 14.818 & 0.027 & -0.063 \\
20061204 & $19: 20: 16.0$ & 540 & 2454074.30574 & 14.840 & 0.028 & -0.040 \\
20061204 & $19: 42: 41.0$ & 540 & 2454074.32131 & 14.822 & 0.026 & -0.026 \\
20061204 & $20: 05: 09.0$ & 540 & 2454074.33691 & 14.809 & 0.026 & -0.009 \\
20061204 & $20: 27: 41.0$ & 540 & 2454074.35256 & 14.840 & 0.027 & 0.006 \\
\hline
\end{tabular}

Note. - This table is available in its entirety in machine-readable and Virtual Observatory (VO) forms in the online journal. A portion is shown here for guidance regarding its form and content. 


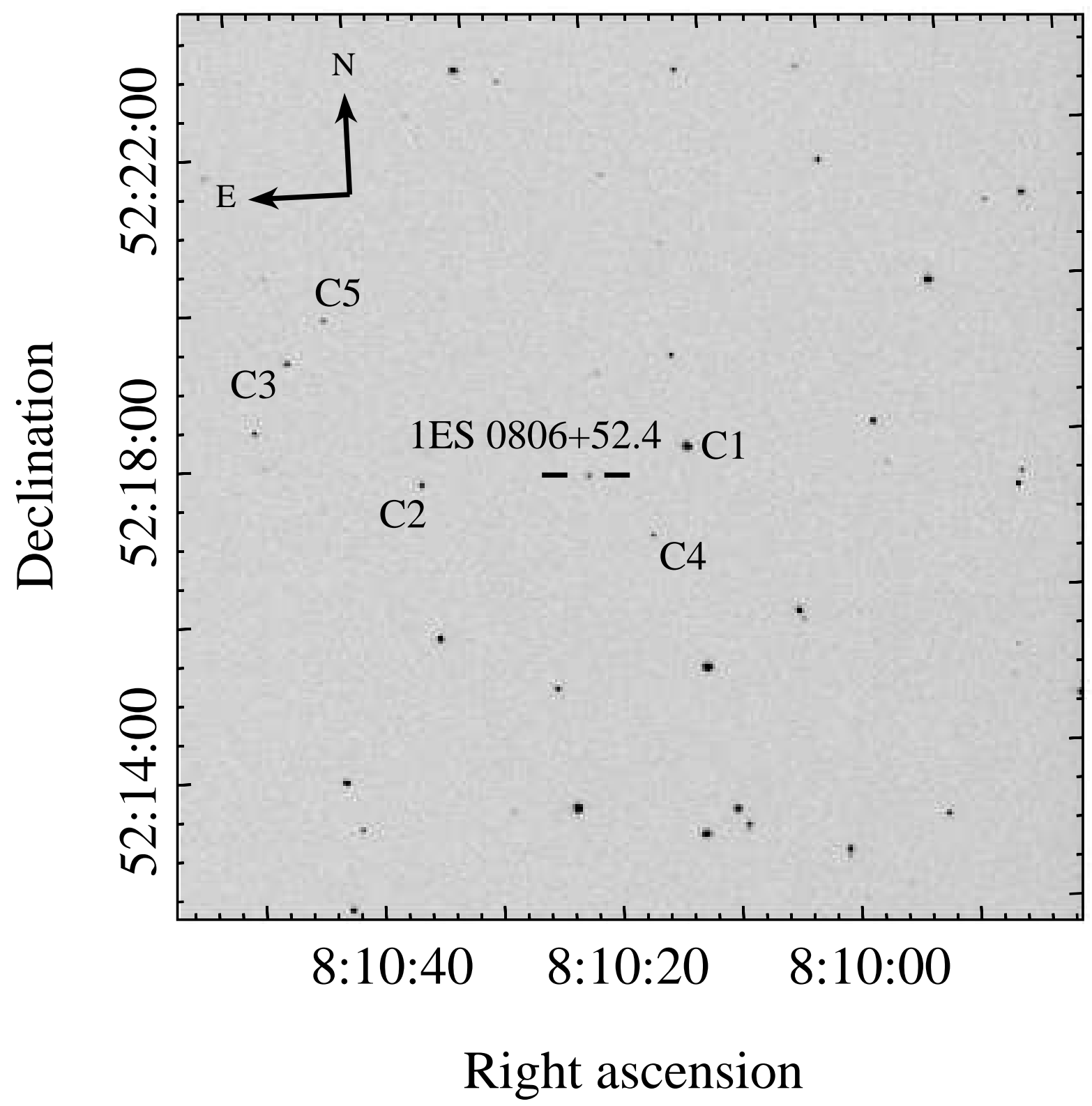

Fig. 1.- Finding chart of 1ES $0806+52.4$ and the 5 comparison stars taken in $i$ band with the $60 / 90$ Schmidt telescope on Feb $5^{t h}, 2007$. The size is $12^{\prime} \times 12^{\prime}$ (or $512 \times 512$ in pixels). 


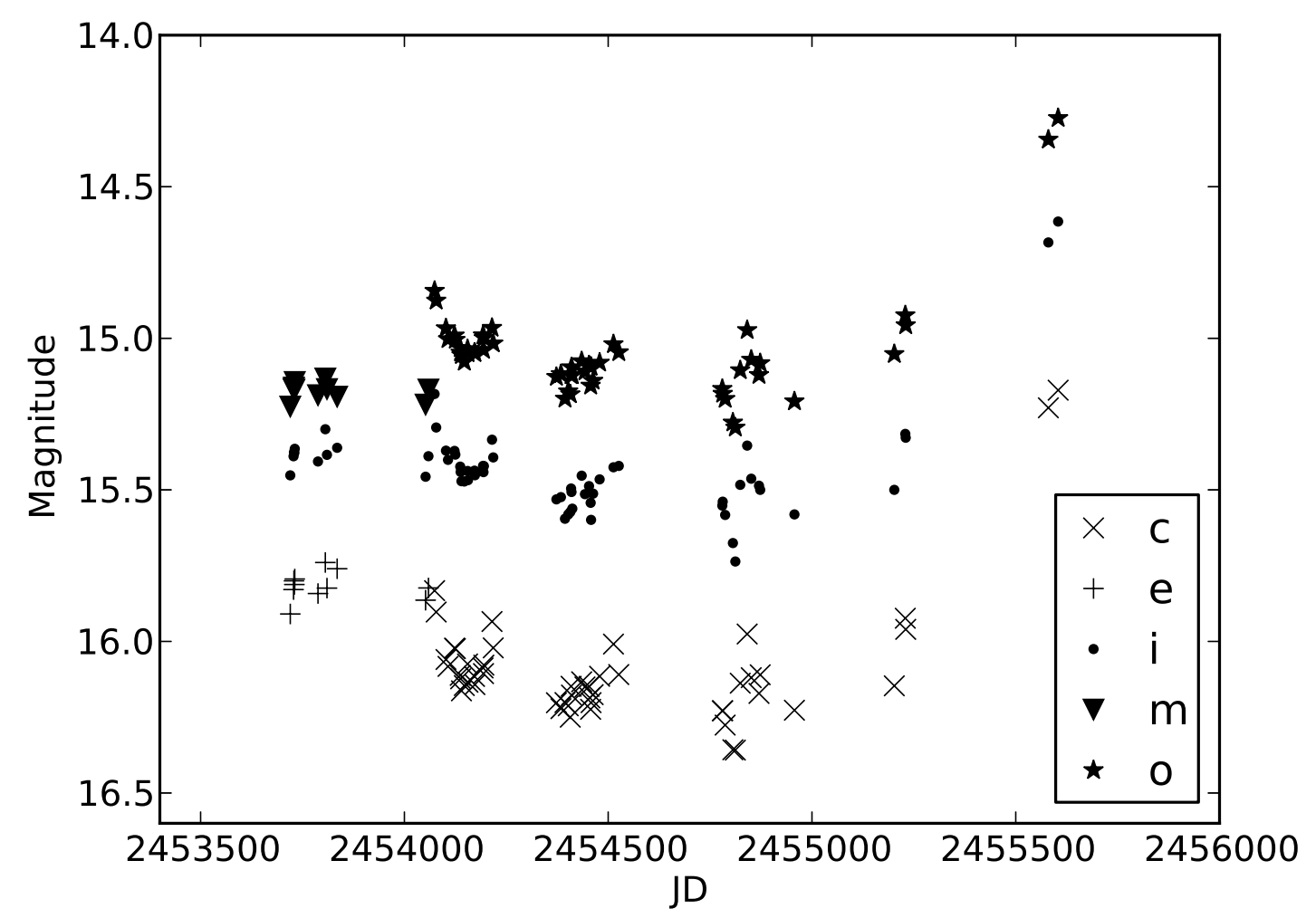

Fig. 2.- Nightly average light curves of $1 \mathrm{ES} 0806+52.4$ in the $c, e, i, m$, and $o$ bands. 

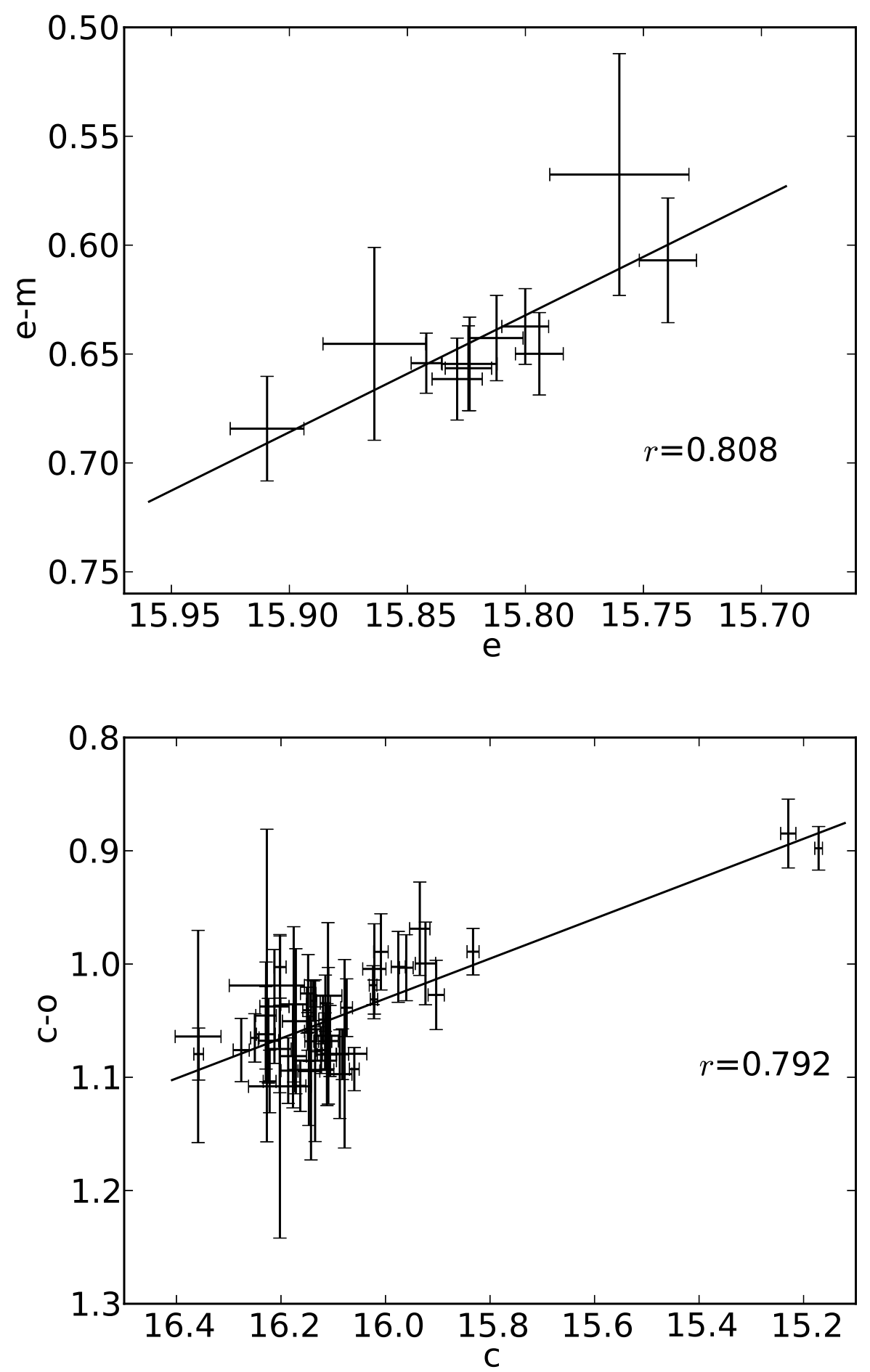

Fig. 3.- Long term color behavior of the first (up) and the second (bottom) stages. The nightly-average data are used. Strong bluer-when-brighter chromatisms can be found. 


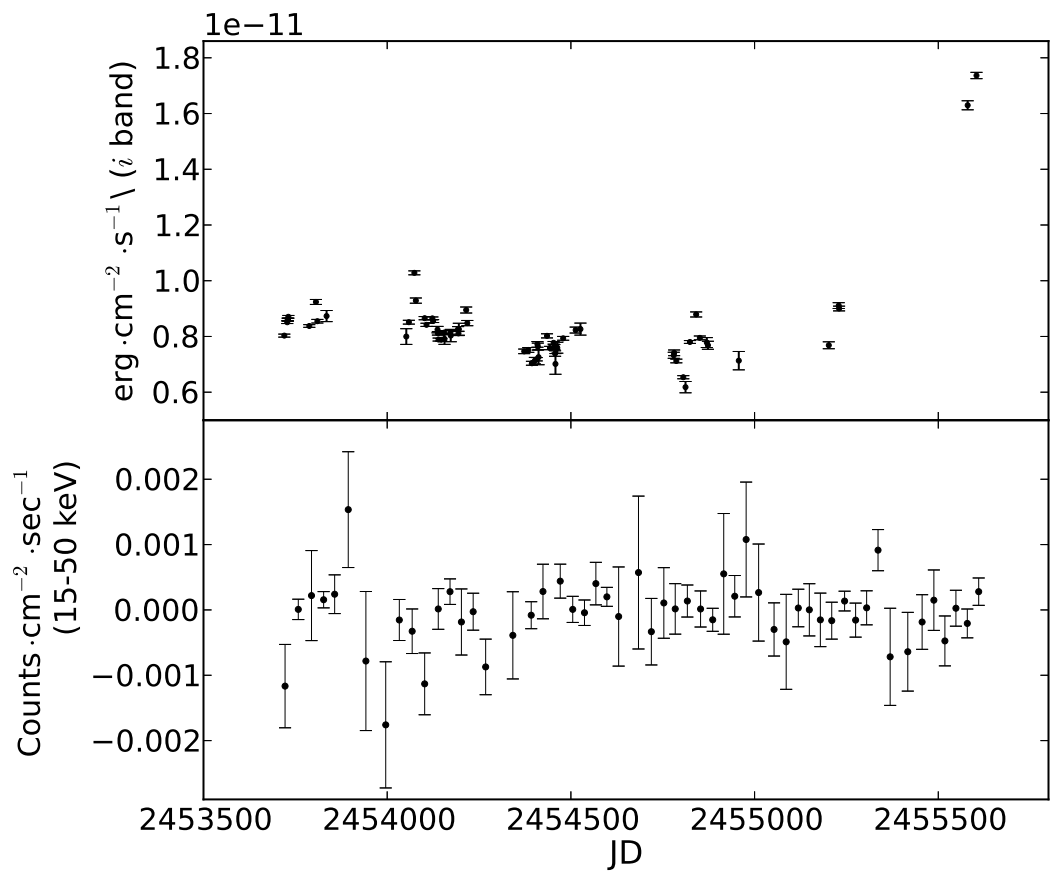

Fig. 4. - Light curve in $i$ band and X-ray band are shown here. Monthly average X-ray data were presented instead of daily average to provide a clearer view of the general variation in the graph. 


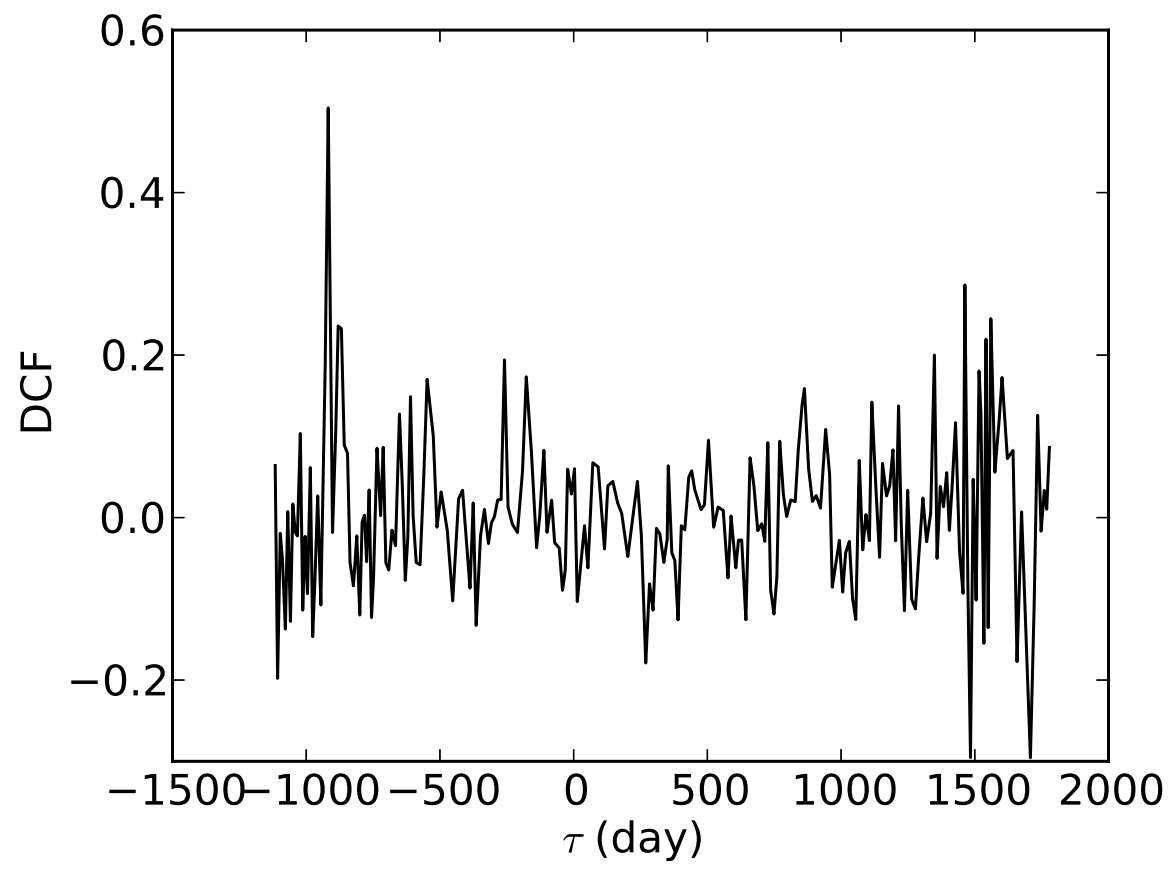

Fig. 5. - The DCF of the z-transformed discrete correlation function. 